\title{
Self-organized criticality of wildfires ecologically revisited
}

\author{
Carlo Ricotta a,*, Margarita Arianoutsou b, Ricardo Díaz-Delgado ${ }^{c}$, Beatriz \\ Duguy d, Francisco Lloret c ${ }^{c}$, Eleni Maroudi ${ }^{b}$, Stefano Mazzoleni ${ }^{\mathrm{e}}$, José \\ Manuel Moreno ${ }^{\mathrm{f}}$, Serge Rambal ${ }^{\mathrm{g}}$, Ramon Vallejo ${ }^{\mathrm{d}}$, Antonio Vázquez ${ }^{\mathrm{f}}$ \\ a Department of Plant Biology, Uniuersity of Rome 'La Sapienza', Piazzale Aldo Moro 5, 00185, Rome, Italy \\ ${ }^{\mathrm{b}}$ Department of Ecology and Systematics, Faculty of Biology, School of Sciences, Uniuersity of Athens, Panepistimiopolis, \\ 15784 Athens, Greece \\ c CREAF, Facultat de Ciències, Uniuersitat Autònoma de Barcelona, Bellaterra, 08193 Barcelona, Spain \\ d Centro de Estudios Ambientales del Mediterraneo, C/Charles Darwin 14, 46980 Paterna, Valencia, Spain \\ e Istituto di Botanica, Facoltà di Agraria, Uniuersità di Napoli, 80055 Portici, Napoli, Italy \\ ${ }^{\mathrm{f}}$ Facultad de Ciencias del Medio Ambiente, Uniuersitad de Castilla-La Mancha, Auenida Carlos III, 45071 Toledo, Spain \\ ${ }^{g}$ DREAM Unit, CEFE-CNRS, 1919 Route de Mende, 34293 Montpellier Cedex 5, France
}

\begin{abstract}
Wildfire cumulative frequency-area distributions of Mediterranean landscapes are examined for agreement with self-similar (fractal) behavior. Our results support landscape-specific restricted scaling regions of 1.5-3.5 orders of magnitude in size, which are delimited by breakpoints or 'cut-offs'. By identifying the extent of such regions in the fractal frequency-area distribution of wildfires, fractal statistics may give a deeper insight into the scale-invariant dynamics of fire spread, whereas the observed cut-offs may be related to changes in the process-pattern interactions that control wildfire propagation at the landscape scale..
\end{abstract}

Keywords: Fractal statistics; Self-organized criticality; Wildfires

\section{Introduction}

Bak et al. (1988) developed the concept of self-organized criticality (SOC) to model the behavior of extended dynamical systems, which per-

\footnotetext{
* Corresponding author. Tel.: +39-06-49912408; fax: + 3906-4457540.

E-mail address: carlo.ricotta@uniroma1.it (C. Ricotta).
}

sistently operate at states of critical equilibrium with no length or time scales others than those deduced from the size of the system and the elementary activation mechanism. SOC was intended to explain the abundance of fractals in nature and has been suggested as a possible explanation for the $1 / f$ noise seen in many frequency size distributions of complex natural phenomena, 
such as earthquakes, avalanches and various ecosystem properties (Bak and Tang, 1989; Sornette and Sornette, 1989; Noever, 1993; Jørgensen et al., 1998).

The paradigmatic SOC model is illustrated by a simple pile of sand built by randomly adding a grain at a time onto a circular platform. The sand pile will grow, increasing its slope until at any position on the pile the slope becomes too steep and some grains will cause a microscopic avalanche falling off the edge of the platform. If we simply continue to add grains, the slope of the pile steepens and the average size of the avalanches becomes larger. The pile stops growing at the critical state, when for every grain that is added to the pile, on average one grain will fall off the edge of the platform. However, the sand grains do not fall off the edge of the platform one at a time, but rather causing avalanches of any size. The cumulative frequency-size distribution for such avalanches gives the characteristic powerlaw:

$N(>S) \vee S^{-\alpha}$

where $N(>S)$ is the number of avalanches with size greater than $S$ and $a$ is the fractal scaling exponent (Bak et al., 1988).

In other words, at the critical state, a large interactive system such as a sandpile follows its own emergent non-linear behavior, which could not be predicted from the dynamics of the individual grains. Such non-linear systems must be necessarily treated statistically without producing specific details. Therefore, robust power-law frequency-size distributions in the form of Eq. (1) are generally considered as the most striking signature of SOC.

Recently, Malamud et al. (1998) and Ricotta et al. (1999) showed that different wildfire time series from different regions and climates exhibit noncumulative landscape-specific power-law frequency-area distributions over many orders of magnitude, and that these distributions are consis- tent with self-organized critical behavior. This is not surprising because wildfires are highly complex events involving climatic, vegetational, and human factors as well as physical and topographic conditions. Thus, frequency and extent of wildfires on a regional basis show non-linear behavior without any simple way to predict specific details.

Nevertheless, the results of Malamud et al. (1998) contrast with the findings of Ricotta et al. (1999) that, like many other ecological data, cumulative frequency - area distributions of wildfire time series are self-similar only over restricted scaling regions. The aim of this paper is thus to emphasize the ecological implications of the observed restricted scaling regions in the frequency area distribution of wildfires and of breakpoints between them.

\section{Data}

We analyzed the frequency - area distributions of eight wildfire time series in highly fragmented and managed Mediterranean regions with very different vegetation types. Due to the severe summer drought typical of Mediterranean regions, most fires occurred during the summer season.

The data include: (a) 1236 fires in Molise (Italy) during 1970-1996; (b) 508 fires in Cilento (Italy) during 1975-1996; (c) 215 fires in the Simbruini Mountains (Italy) during 1970-1997; (d) 2105 fires in Sierra de Gredos (Spain) during 19741999; (e) 132 fires in the Alicante region during 1973-1996; (f) 204 fires in the Ribera d'Ebre (Spain) during 1983-1997; (g) 335 fires in the Venaco region (Corse, France) during 1977-1997; and (h) 336 fires on Mount Penteli (Greece) during 1954-1996. The data sets are available from the authors upon request. Double-log cumulative frequency-area distributions for the eight data sets are presented in Fig. 1.

Fig. 1. Cumulative frequency -area distributions for the data sets of Molise (A), Cilento (B), Simbruini Mountains (C), Sierra de Gredos (D), Alicante region (E), Ribera d'Ebre (F), Venaco region (G), and Mount Penteli (H). For each data set, the number of fires $N(>S)$ with size greater than $S$ is plotted as a function of $S$ in a double-log space. 

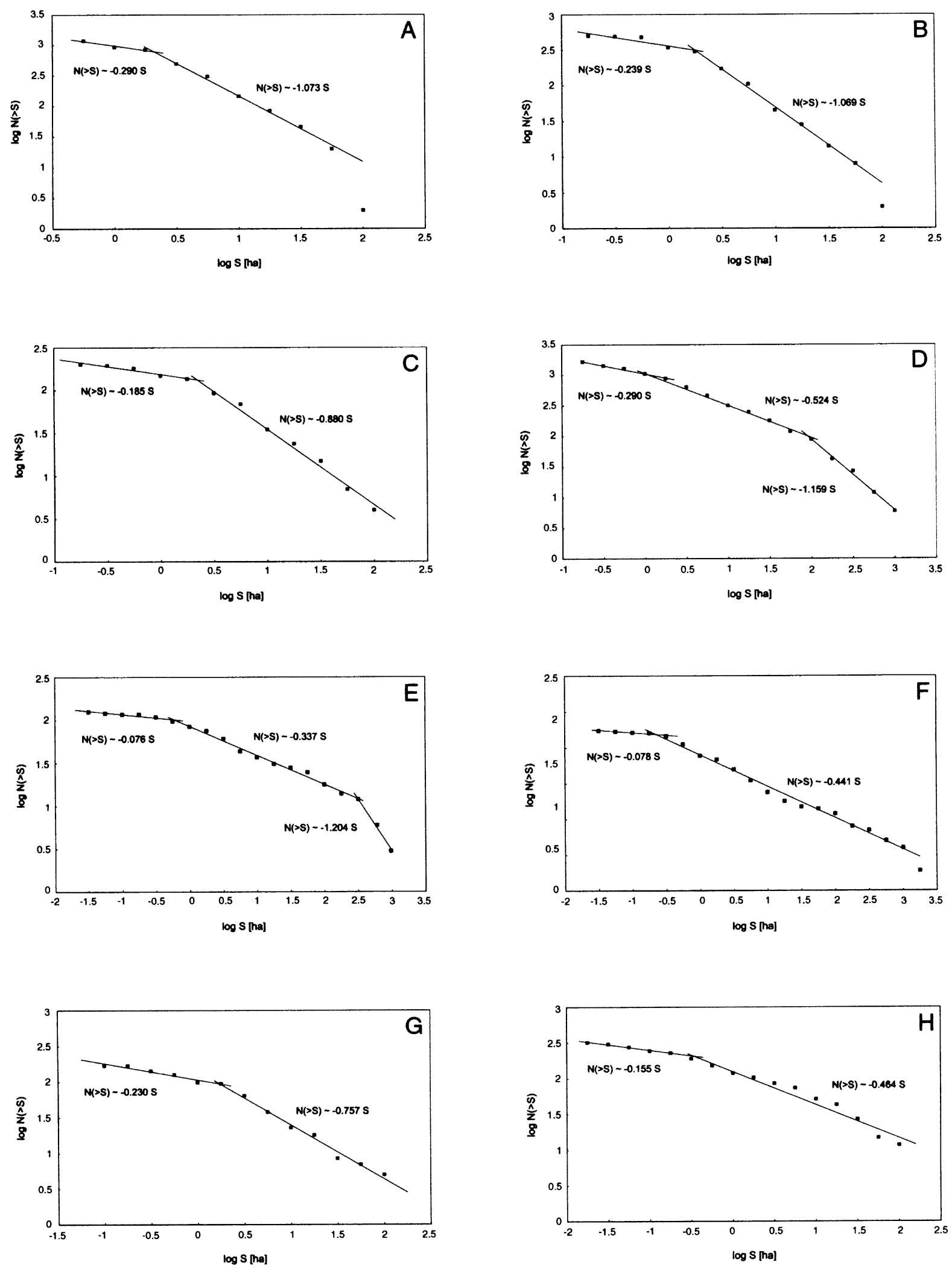

Fig. 1. (Continued) 


\section{Results and discussion}

As shown in Fig. 1, wildfires spanned up to five orders of magnitude in size. Restricted power-law behavior can be seen to describe wildfire frequency - area distributions adequately across a restricted scaling range of 1.5-3.5 orders of magnitude in size delimited by breakpoints or 'cut-offs'. Within each power-law region, the landscape-specific fractal scaling exponent a (i.e. the slope of the line segment in the double-log space) is close to constant suggesting that scale-invariant dynamics generated the observed frequency -area distribution. At both ends, different power-law regions equally describe the frequency-area distributions of smaller and larger fires.

The observed cut-offs were previously attributed to incomplete data and unreliable statistics at both ends of the frequency-area distributions and to extinction efforts, especially at the smaller forest fire areas (Ricotta et al., 1999; Malamud, personal communication).

However, it is generally agreed that large fires are controlled by one set of environmental variables, such as wind velocity and direction, whereas smaller fires are most likely driven by another set of variables, such as local variations in fuel load and moisture, topography and landscape fragmentation (Pyne, 1984; Johnson, 1992; Bessie and Johnson, 1995). In addition, one of the tenets of ecological hierarchy theory is that, for hierarchically structured landscapes, landscape spatial heterogeneity shows different patterns at different scales (Ricklefs, 1987; Kotliar and Wiens, 1990; O'Neill et al., 1991; Holling, 1992). The cut-offs may therefore reflect more or less abrupt changes in the landscape-specific process-pattern interactions that control wildfire propagation, rather than statistical inaccuracies.

This effect is not new in ecological literature and has been shown to hold for a variety of other perturbation effects (Krummel et al., 1987; Milne, 1988; Meltzer and Hastings, 1992). It is well established that similar restricted scaling regimes appear unavoidable in all natural systems where fractal dynamics plays a constructive role. In other words, all natural systems are only loose fractals (Hastings and Sugihara, 1994).
However, due to the relatively short periods of observation (varying between 43 and 15 years for the Mount Penteli and the Ribera d'Ebre data bases, respectively), the location of the upper cutoffs in the analyzed wildfire frequency -area distributions may also be biased by the limited number of very large wildfires with long repeat times and, consequently, very small frequencies.

Nevertheless, although still incapable of approaching five orders in magnitude, the fractal behavior of wildfire statistics gives new insights into a basic question in fire-related ecological studies (see Turner et al., 1994). Since differences in the physics of wildfires influence the post-fire spatial and temporal dynamics of plant recolonization (Romme and Knight, 1981; Malanson, 1984; Turner et al., 1997), determining the landscape-specific range of scales over which wildfire frequency - area distributions exhibit self-similarity may be of some significance for understanding whether large fires are simply quantitatively larger than small fires, or if they exhibit some qualitative difference that emerge after crossing some threshold in size.

\section{Acknowledgements}

This research was conducted within the LUCIFER Project (Land Use Change Interactions with Mediterranean Landscapes). Financial support was provided by the DG XII-Environment and Climate Program under the Fourth Framework Program of the European Union (ENV-CT960320).

\section{References}

Bak, P., Tang, C., Wiesenfeld, K., 1988. Self-organized criticality. Phys. Rev. A 38, 364-374.

Bak, P., Tang, C., 1989. Earthquakes as a self-organized critical phenomenon. J. Geophys. Res. B 94, 15635-15637.

Bessie, W.C., Johnson, E.A., 1995. The relative importance of fuels and weather on fire behavior in subalpine forests. Ecology 76, 742-762.

Johnson, E.A., 1992. Fire and Vegetation Dynamics: Studies from the North American Boreal Forest. Cambridge University Press, Cambridge. 
Hastings, H.M., Sugihara, G., 1994. Fractals: A Users Guide for the Natural Sciences. Oxford University Press, Oxford.

Holling, C.S., 1992. Cross-scale morphology, geometry and dynamics of ecosystems. Ecol. Monogr. 62, 447-502.

Jørgensen, S.E., Mejer, H., Nielsen, S.N., 1998. Ecosystem as self-organizing critical systems. Ecol. Model. 111, 261-268.

Kotliar, N.B., Wiens, J.A., 1990. Multiple scales of patchiness and patch structure: a hierarchical framework for the study of heterogeneity. Oikos 59, 253-260.

Krummel, J.R., Gardner, R.H., Sugihara, G., O'Neill, R.V., Coleman, P.R., 1987. Landscape patterns in a disturbed environment. Oikos 48, 321-324.

Malamud, B.D., Morein, G., Turcotte, D.L., 1998. Forest fires: an example of self-organized critical behavior. Science 281, 1840-1842.

Malanson, G.P., 1984. Intensity as a third factor of disturbance regime and its effect on species diversity. Oikos 43, 411-413.

Meltzer, M.I., Hastings, H.M., 1992. The use of fractals to assess the ecological impact of increased cattle population: case study from the Runde Communal Land, Zimbabwe. J. Appl. Ecol. 29, 635-646.

Milne, B.T., 1988. Measuring the fractal geometry of landscapes. Appl. Math. Comput. 27, 67-79.
Noever, D.A., 1993. Himalayan sandpiles. Phys. Rev. E 47, 724-725.

O’Neill, R.V., De Angelis, D.L., Allen, T.F.H., 1991. A Hierarchical Concept of Ecosystems. Princeton University Press, Princeton, NJ.

Pyne, S.J., 1984. Introduction to Wildland Fire. Wiley \& Sons, New York.

Ricklefs, R.E., 1987. Community diversity: relative roles of local and regional processes. Science 235, 167-171.

Ricotta, C., Avena, G.C., Marchetti, M., 1999. The flaming sandpile: self-organized criticality and wildfires. Ecol. Model. 119, 73-77.

Romme, W.H., Knight, D.H., 1981. Fire frequency and subalpine forest succession along a topographic gradient in Wyoming. Ecology 62, 319-326.

Sornette, A., Sornette, D., 1989. Self-organized criticality and earthquakes. Europhys. Lett. 9, 197-202.

Turner, M.G., Hargrove, W.W., Gardner, R.H., Romme, W.H., 1994. Effects of fire on landscape heterogeneity in Yellowstone National Park, Wyoming. J. Veg. Sci. 5, 731-742.

Turner, M.G., Romme, W.H., Gardner, R.H., Hargrove, W.W., 1997. Effects of fire on early succession in Yellowstone National Park. Ecol. Monogr. 67, 411-433. 